\title{
Diferença de tempo de positividade: método útil no diagnóstico de infecção de corrente sanguinea relacionada com cateter?
}

\author{
Differential time to positivity: a useful tool for the diagnosis of catheter-related infections?
}

Karoline de Lemes Giuntini Corrêa'; Gisele Madeira Duboc de Almeida²; João Nobrega de Almeida Júnior³; Flávia Rossi

\section{unitermos \\ Infecção de corrente sanguínea relacionada com cateter \\ Diferença de tempo de positividade \\ Cateter venoso central \\ Infecção hospitalar}

\section{resumo}

Introdução: As infecções de corrente sanguínea relacionadas com cateter (ICSRCs) apresentam impacto significativo na morbidade e na mortalidade de pacientes internados, além de elevar custos hospitalares. A utilização de equipamentos automatizados no processamento de hemoculturas gerou uma alternativa para diagnóstico de ICSRC por meio da análise da diferença de tempo de positividade (DTP) entre hemoculturas pareadas (coletadas simultaneamente) de sangue periférico e sangue de cateter. Um diagnóstico acurado e rápido dessas infecções pode otimizar as condutas clínicas e terapêuticas, poupando a retirada precoce dos cateteres. Objetivos: Avaliar na rotina a DTP como ferramenta auxiliar no diagnóstico de ICSRC e determinar os principais microrganismos isolados. Métodos: Foram avaliadas retrospectivamente hemoculturas coletadas no complexo do Hospital das Clínicas da Faculdade de Medicina da Universidade de São Paulo (HC/FMUSP) de maio a agosto de 2008. Somente amostras que apresentaram DTP maior que 120 minutos foram consideradas possíveis ICSRCs pelo critério laboratorial. Resultados: A seção processou 11.017 hemoculturas aeróbias durante o período de estudo; somente $5 \%$ foram coletadas de forma pareada. Destas, 148 (28\%) foram positivas, sendo $9 \%$ com crescimento somente em sangue periférico, $41 \%$ somente em sangue de cateter e $50 \%$ em ambas as amostras com $88 \%$ de homologia de microrganismos identificados. A DTP apresentou valores acima de 120 minutos em $50 \%$ dos casos e os microrganismos mais isolados foram Staphylococcus aureus (22\%), Candida spp. (18\%), Klebsiella spp. (7\%) e Enterobacter spp. (7\%). Conclusão: A determinação da DTP como ferramenta auxiliar no diagnóstico de ICSRC é viável e fácil de ser executada em laboratórios de rotina com automação, porém o processo de coleta das amostras pareadas deve ser rigidamente padronizado.

\section{abstract}

Introduction: Not only do catheter related bloodstream infections (CRBSIs) have considerable impact on morbidity and mortality in hospitalized patients, but they also raise hospital costs. The use of automated equipment in blood culture processing has allowed an alternative diagnosis of CRBSI by analyzing the differential time to positivity (DTP) of paired blood cultures (collected simultaneously) of peripheral blood and catheter blood. A rapid and accurate diagnosis of these infections may optimize clinical and therapeutic management, which prevents early catheter removal. Objectives: To assess DTP as an auxiliary tool for the diagnosis of CRBSI as well as to determine the main isolated microorganisms. Methods: We evaluated blood cultures that had previously been collected in the complex Hospital das Clínicas da Faculdade de Medicina da Universidade de São Paulo (HC/FMUSP) from May to August 2008. According to the laboratory criteria, only DTP higher than 120 minutes was regarded as possible CRBSI. Results: During the investigation period 11,017 aerobic blood cultures were processed, from which only 5\% were paired samples. One hundred forty-eight (28\%) samples were positive, from which 9\% showed growth in peripheral blood, $41 \%$ only in catheter blood and $50 \%$ in both blood samples with $88 \%$ homology of identified microorganisms. DTP higher than 120 minutes occurred in $50 \%$ of the cases. The most common isolated microorganisms were: Staphylococcus aureus (22\%), Candida spp. (18\%), Klebsiella spp (7\%). and Enterobacter spp (7\%). Conclusion: The determination of the DTP as an auxiliary tool for the diagnosis of CRBSI is feasible and easily performed in clinical laboratories with automation, although the process of paired sample collection must be rigidly standardized. key words

Catheter related bloodstream infections

Differential time to positivity

Central venous catheter

Hospital infections

1. Farmacêutica bioquímica; biologista da Seção de Microbiologia da Divisão de Laboratório Central do Hospital das Clínicas da Faculdade de Medicina da Universidade de São Paulo (DLC-HC-FMUSP).

2. Médica; infectologista da Seção de Microbiologia da DLC-HC-FMUSP.

3. Médico; infectologista da Seção de Microbiologia da DLC-HC-FMUSP.

4. Médica; diretora técnica da Seção de Microbiologia da DLC-HC-FMUSP. 


\section{Introdução}

Os dispositivos intravasculares foram introduzidos na prática clínica em meados de 1940 e atualmente são indispensáveis na assistência à saúde de inúmeros pacientes, particularmente aqueles internados em unidades de terapia intensiva (UTI) ou sob quimioterapia ${ }^{(10,28,30)}$. Esses acessos intravasculares são utilizados para monitoramento hemodinâmico, realização de hemodiálise, nutrição parenteral, administração de hemoderivados e medicamentos.

Aproximadamente 15 milhões de cateteres venosos centrais (CVCs) são utilizados por ano nos EUA em UTIs ${ }^{(11,14,17) .}$

A taxa de todas as infecções relacionadas com cateter (IRCs) é de difícil determinação, já que seus índices são influenciados por fatores associados ao paciente (como severidade da doença), ao cateter e institucionais. Entre os hospitais participantes do National Healthcare Safety Network (NHSN), em 2006, a taxa de infecção de corrente sanguínea relacionada com cateter (ICSRC) variou de 1,3/1.000 cateteres-dia em unidade de internação a 5,6/1.000 cateteres-dia em UTI de queimados ${ }^{(13,14)}$. Um estudo realizado no Hospital das Clínicas da Faculdade de Medicina da Universidade de São Paulo (HC/FMUSP), publicado em 2005, referiu que a ICSRC foi a mais importante infecção hospitalar nas UTIs dessa instituição no período em pesquisa, com taxas quatro vezes maiores que o $\mathrm{NHSN}^{(14)}$.

São vários os fatores de risco associados ao aumento da ICSRC. Os intrínsecos ao paciente são alteração do estado imunológico, extremos de idade, preexistência de comorbidades, gravidade da doença, má nutrição e colonização da pele. Já os extrínsecos podem ser específicos quanto ao tipo de cateter ou comum a todos. Os principais fatores relacionados com os CVCs são local de inserção, presença ou ausência de túnel, número de lumens, uso de sistema de monitorização de pressão, infusão de nutrição parenteral, tempo de permanência do cateter, tipo de cateter, dificuldade de inserção, local de internação e colonização do canhão. Além desses fatores de risco, podemos citar outros que são independentes para ICSRC, como hospitalização prolongada antes da cateterização e deficiências de cuidado com cateter ${ }^{(13,14,28)}$.

As infecções de corrente de sanguínea (ICSs) apresentam impacto significativo na morbidade e na mortalidade, além de elevar os custos hospitalares ${ }^{(11,17)}$. Estima-se que nos EUA ocorram de 200 a 400 mil ICSs por ano e a maioria está relacionada com o uso de acessos intravasculares. Acredita-se que as IRCs sejam responsáveis por $10 \%$ a $20 \%$ das infecções hospitalares ${ }^{(8,16,30)}$.
As complicações associadas a essas infecções são importantes, uma vez que a mortalidade relacionada com a IRC varia de $12 \%$ a $30 \%(8,13,19,24,30)$. Além disso, a literatura relata que, por ano, nos EUA, aproximadamente 80 mil CVCs são infectados, trazendo custo adicional para a saúde de U $\$ 60$ a 460 milhões, uma vez que esses pacientes permanecem mais tempo internados e necessitam de procedimentos adicionais (cerca de 10 a 40 dias) ${ }^{(4,9,13,20)}$.

A patogênese da ICSRC é complexa e multifatorial. Os microrganismos podem ter acesso ao dispositivo intravascular por meio da via extraluminal do cateter, quando organismos da microbiota local migram através do trato percutâneo ou, por contaminação no momento de manipulação do CVC, principalmente pela equipe de saúde. $\mathrm{O}$ acesso pela via intraluminal do cateter pode ocorrer por contaminação do fluido de infusão, contaminação da conexão por manipulação pela equipe de saúde ou por microrganismos carreados pela

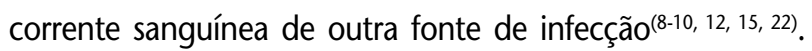
A via de colonização extraluminal a partir de organismos da microbiota da pele parece ser a principal via de infecção em CVC de curta duração, enquanto para CVC de longa duração apresenta-se a via intraluminal de colonização ${ }^{(4,8,9,16)}$.

Os microrganismos que comumente causam ICS adquirida no ambiente hospitalar mudaram ao longo do tempo. Durante 1986-1989, o Staphylococcus spp. coagulase negativa (SCN), seguido por Staphylococcus aureus, foram as mais frequentes causas de ICS, representando $27 \%$ e $16 \%$ respectivamente. Dados de 1992 a 1999 indicam que SCN e Enterococcus spp. eram as causas mais comuns de ICS adquirida no ambiente hospitalar. Hoje, os patógenos mais constantes da ICS no ambiente hospitalar são $\operatorname{SCN}(31,3 \%)$, S. aureus (20,2\%), Enterococcus spp. (9,4\%) e Candida spp. (9\%). Os bacilos Gram-negativos ocorrem entre 19\% e $21 \%$ das ICSRCs $(8,9,13,14,16)$.

Para todos os patógenos que causam ICSRC, a resistência a antimicrobianos é um problema, principalmente nas UTIs. Mais de $50 \%$ dos S. aureus, isolados em UTIs, são resistentes à oxacilina, contudo a incidência em ICRSC tem diminuído nos últimos anos, possivelmente como resultado dos esforços de prevenção. Bacilos Gram-negativos resistentes a cefalosporinas de terceira geração e carbapenens, assim como Candida spp. resistente a fluconazol, estão cada vez mais comuns ${ }^{(13,14,21,25-27)}$.

O diagnóstico clínico de IRC mostra-se difícil e complexo, pois os sinais e sintomas presentes possuem baixa especificidade, além de ser prejudicado pela falta de um método gold standard, que diferencie contaminação, 
colonização e infecção ${ }^{(16)}$. Atualmente, a metodologia mais utilizada para avaliar ICSRC é a cultura semiquantitativa de ponta de cateter ${ }^{(15)}$ associada à hemocultura qualitativa periférica. Dados da literatura utilizando essa metodologia apontam, no entanto, para uma remoção desnecessária de $75 \%$ a $85 \%$ dos cateteres em pacientes febris, dos quais somente $15 \%$ a $25 \%$ dos dispositivos provam realmente estar infectados $(4,21,30)$.

Dos métodos diagnósticos disponíveis de rotina para ICSRC que preservam o cateter, a diferença de tempo de positividade (DTP) e a hemocultura pareada quantitativa de sangue periférico e sangue de cateter são descritas, porém pouco aplicadas na rotina ${ }^{(5,6,24)}$.

A utilização de equipamentos automatizados no processamento de hemoculturas gerou uma alternativa para o diagnóstico de ICSRC sem que haja necessidade de remoção do cateter. A análise da DTP baseia-se na diferença de tempo entre hemoculturas positivas de sangue periférico e sangue de cateter coletadas com intervalo máximo de 15 minutos e com volumes iguais ${ }^{(2,3,15,23,24)}$. Para diferenças $\geq 120$ minutos entre o tempo de positividade (TP) de hemocultura central e periférica, interpreta-se como possível ICSRC, lembrando que o microrganismo isolado deve ser o mesmo em ambas as hemoculturas ${ }^{(3,23)}$. Equipamentos automatizados de hemoculturas fazem leituras dos frascos em intervalos curtos e contínuos de tempo, possibilitando, desse modo, determinar a curva de crescimento do microrganismo e gerando um gráfico de positividade de fácil acompanhamento. Essa técnica, porém, apresenta limitações na fase pré-analítica, que deve ser muito bem padronizada, e também quando o antibiótico é administrado antes da coleta de sangue pelo acesso venoso central $(6,23)$.

Vários estudos têm avaliado o potencial da DTP como ferramenta no diagnóstico de ICSRC. Raad et al. estabeleceram que a DTP com valores $\geq 120$ minutos apresentava sensibilidade de $81 \%$ e especificidade de $92 \%$ para CVC de curta duração, com valores de $93 \%$ e $73 \%$ para CVC de longa duração(23).

Em nossa instituição, a determinação da DTP tem sido solicitada, mas ainda não há uma padronização da técnica na fase pré-analítica.

\section{Objetivos}

Os objetivos principais deste trabalho foram avaliar a aplicação da detecção laboratorial da DTP em hemoculturas coletadas de rotina e, posteriormente, determinar os principais microrganismos nos pares com DTP acima de 120 minutos.

\section{Métodos}

Realizou-se um estudo retrospectivo observacional descritivo relacionado com hemoculturas provenientes de pacientes internados no complexo do HC/FMUSP, de maio a agosto de 2008. As hemoculturas foram coletadas a critério clínico, conforme orientado no manual de coleta do laboratório, sendo os frascos enviados à Seção de Microbiologia da Divisão de Laboratório Central (DLC) do HC/FMUSP e processados no sistema Bactec ${ }^{\circledR}$ 9.240. Os frascos foram incubados a $35^{\circ} \mathrm{C}$ por até cinco dias sob agitação contínua. Esse equipamento automatizado permite monitoramento contínuo e simultâneo de amostras de sangue para detecção de bactérias e fungos. O princípio da tecnologia tem como base a detecção da produção de $\mathrm{CO}_{2}$ resultante do metabolismo microbiano por meio de luz fluorescente. As leituras de fluorescência são realizadas a cada 10 minutos e permitem a construção de gráficos automáticos de monitoramento do crescimento ou não de microrganismos. Amostras positivas têm o TP registrado, possibilitando, desse modo, o cálculo da DTP em hemoculturas coletadas de forma pareada.

A partir dos registros do sistema de informação de gestão hospitalar, foram identificadas as amostras pareadas nesse período. Aquelas que apresentaram homologia de microrganismos e coletadas em um intervalo não superior a 15 minutos foram analisadas graficamente, sendo o resultado da DTP calculado manualmente. A Figura 1 ilustra os gráficos gerados pelo Bactec ${ }^{\circledR}$.

O critério laboratorial utilizado para ICSRC foi um DTP $\geq 120$ minutos, segundo Blot et al. ${ }^{(3)}$. A identificação dos microrganismos foi realizada por meio de provas bioquímicas convencionais e pelo sistema automatizado Vitek $2^{\circledR}$ (bioMérieux) e os testes de sensibilidade aos antimicrobianos foram realizados pela técnica de disco-difusão (Bauer e Kirby, 1966) e pelo método automatizado Vitek $2^{\circledR(7,18)}$.

A cultura de ponta de cateter de pacientes com DTP acima de 120 minutos foi analisada, quando disponível, a fim de verificar positividade e possível homologia de microrganismos com as amostras pareadas, em um intervalo de até oito dias entre as culturas. 


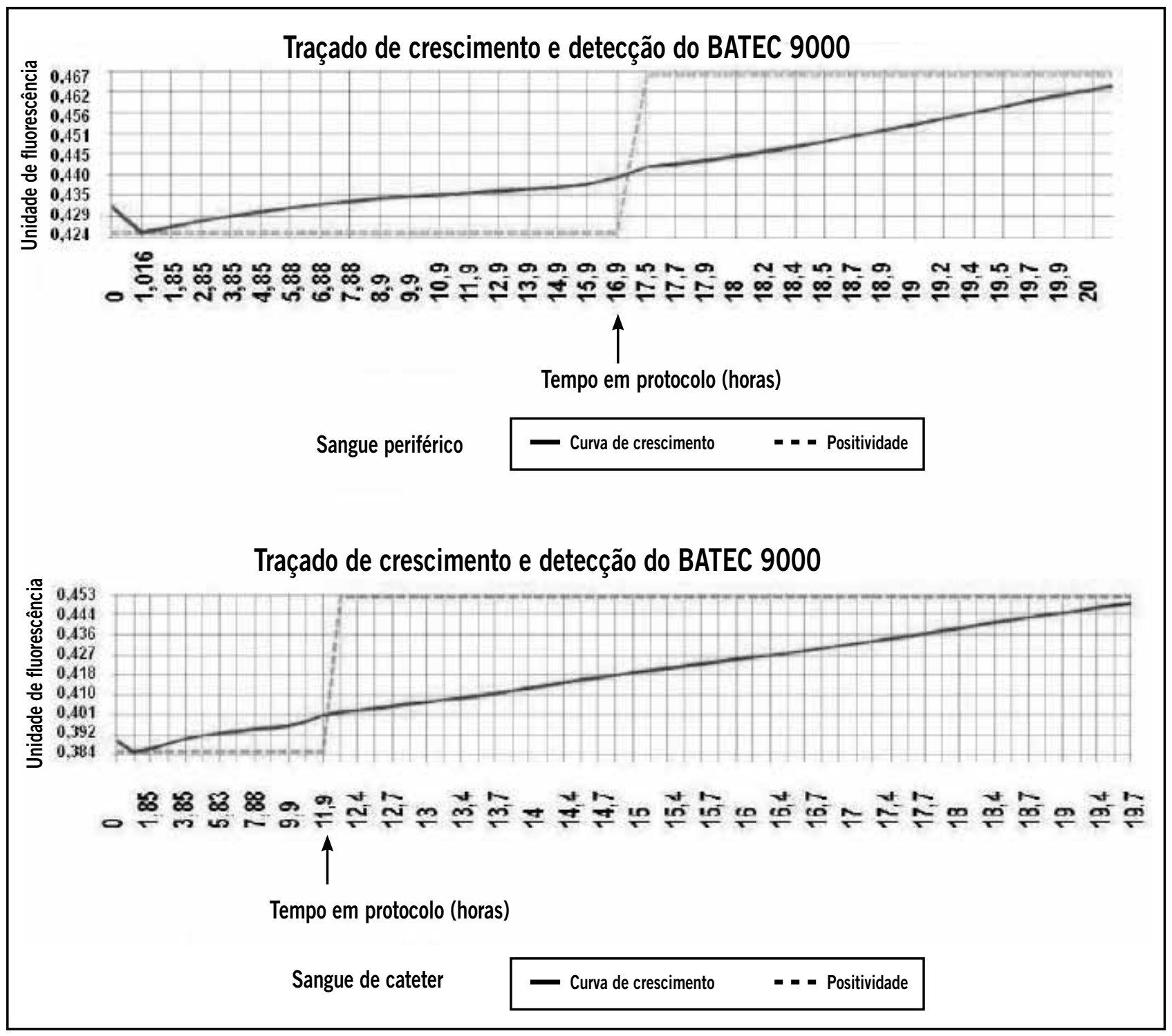

Figura 1 - Ilustração dos dados utilizados para cálculo da DTP

DTP = TP de sangue periférico - TP de sangue de cateter; DTP = 16,9 - 11,9; DTP $=5$ horas $=300$ minutos.

DTP: diferença de tempo de positividade; TP: tempo de positividade.

\section{Resultados}

Durante o período de estudo (maio a agosto de 2008), a Seção de Microbiologia processou 11.017 hemoculturas aeróbias provenientes de pacientes internados no complexo HC/FMUSP. Destas, somente 525 (5\%) foram identificadas como hemoculturas pareadas de sangue de cateter e sangue periférico (C/P), constituindo, assim, a amostra avaliada. Das amostras pareadas, 148 (28\%) foram positivas. Destas, 13 (9\%) apresentaram crescimento microbiano somente em amostras do sangue periférico $(\mathrm{C}-/ \mathrm{P}+), 61(41 \%)$ apresentaram crescimento somente em sangue de cateter ( $\left.\mathrm{C}_{+} / \mathrm{P}_{-}\right)$e $74(50 \%)$ apresentaram crescimento em ambas $\left(\mathrm{C}_{+} / \mathrm{P}+\right)$, sendo que $88 \%$ tiveram homologia de microrganismos isolados (Figura 2). $\mathrm{Na}$ análise das amostras positivas pareadas, nove foram excluídas devido à coleta em horários divergentes. Nos 56 pares de hemocultura em que o cálculo de DTP foi realizado, 28 (50\%) tiveram DTP $\geq 120$ minutos.

A distribuição de microrganismos isolados de acordo com origem, sangue de cateter, sangue periférico e DTP podem ser observadas na Tabela.

A análise do banco de dados laboratoriais mostrou que em 19 (69\%) pacientes com DTP > 120 minutos o cateter foi retirado e enviado para cultura, sendo que somente quatro (22\%) apresentaram cultura positiva com homologia de microrganismo isolado na hemocultura pareada. 


\section{Distribuição de microrganismos isolados de hemoculturas de acordo com origem (sangue de cateter e} Tabela sangue periférico) e DTP

\begin{tabular}{|c|c|c|c|c|c|c|}
\hline \multirow{2}{*}{ Microrganismos } & \multicolumn{2}{|c|}{$\mathrm{C}+/ \mathrm{P}+$} & $\mathrm{C}+/ \mathrm{P}_{+}$ & $C+/ P$ & $\mathrm{C}-\mathrm{P}+$ & Total \\
\hline & DTP $<120$ & DTP $>120$ & DTP não calculada* & & & \\
\hline$n(\%)$ & $28(100)$ & $28(100)$ & $33(100)$ & $65(100)$ & $13(100)$ & $167(100)$ \\
\hline \multicolumn{7}{|c|}{ Gram-positivos } \\
\hline $\begin{array}{l}\text { Staphylococcus coagulase } \\
\text { negativo }\end{array}$ & $\varnothing$ & $2(7)$ & $6(18)$ & $33(51)$ & $4(31)$ & $45(28)$ \\
\hline Staphylococcus aureus & $8(28)$ & $6(22)$ & $7(22)$ & $4(6)$ & $2(17)$ & $27(16)$ \\
\hline Enterococcus spp. & $2(7)$ & $4(14)$ & $5(15)$ & $5(8)$ & $1(7)$ & $17(10)$ \\
\hline \multicolumn{7}{|c|}{ Gram-negativos } \\
\hline Pseudomonas aeruginosa & $3(11)$ & $\varnothing$ & $4(12)$ & $3(4)$ & $4(31)$ & $15(9)$ \\
\hline Acinetobacter spp. & $3(11)$ & $\varnothing$ & $3(9)$ & $\varnothing$ & $1(7)$ & $12(7)$ \\
\hline Escherichia coli & $2(7)$ & $\varnothing$ & $\varnothing$ & $2(3)$ & - & - \\
\hline Klebsiella spp. & $2(7)$ & $2(7)$ & $2(6)$ & $\varnothing$ & $1(7)$ & $4(4)$ \\
\hline Enterobacter spp. & $2(7)$ & $2(7)$ & $\varnothing$ & $\varnothing$ & $\varnothing$ & $6(3)$ \\
\hline Cryseobacterium spp. & $\varnothing$ & $2(7)$ & $2(6)$ & $\varnothing$ & $\varnothing$ & $7(4)$ \\
\hline Outros & $5(18)$ & $5(18)$ & $2(6)$ & $13(20)$ & - & 22 (13) \\
\hline \multicolumn{7}{|c|}{ Levedura } \\
\hline Candida spp. & $1(3)$ & $5(18)$ & $2(6)$ & $1(2)$ & $\varnothing$ & $9(6)$ \\
\hline
\end{tabular}

C+/P+: hemocultura pareada positiva para sangue de cateter e sangue periférico; DTP: diferença de tempo de positividade; *: hemoculturas pareadas excluídas do cálculo de DTP (divergência de microrganismo isolado, horário inadequado de coleta e falta de gráfico de positividade); C+/P-: hemocultura pareada positiva somente para sangue de cateter; C-/P+: hemocultura pareada positiva somente para sangue periférico; $\emptyset$ : microrganismo não isolado.

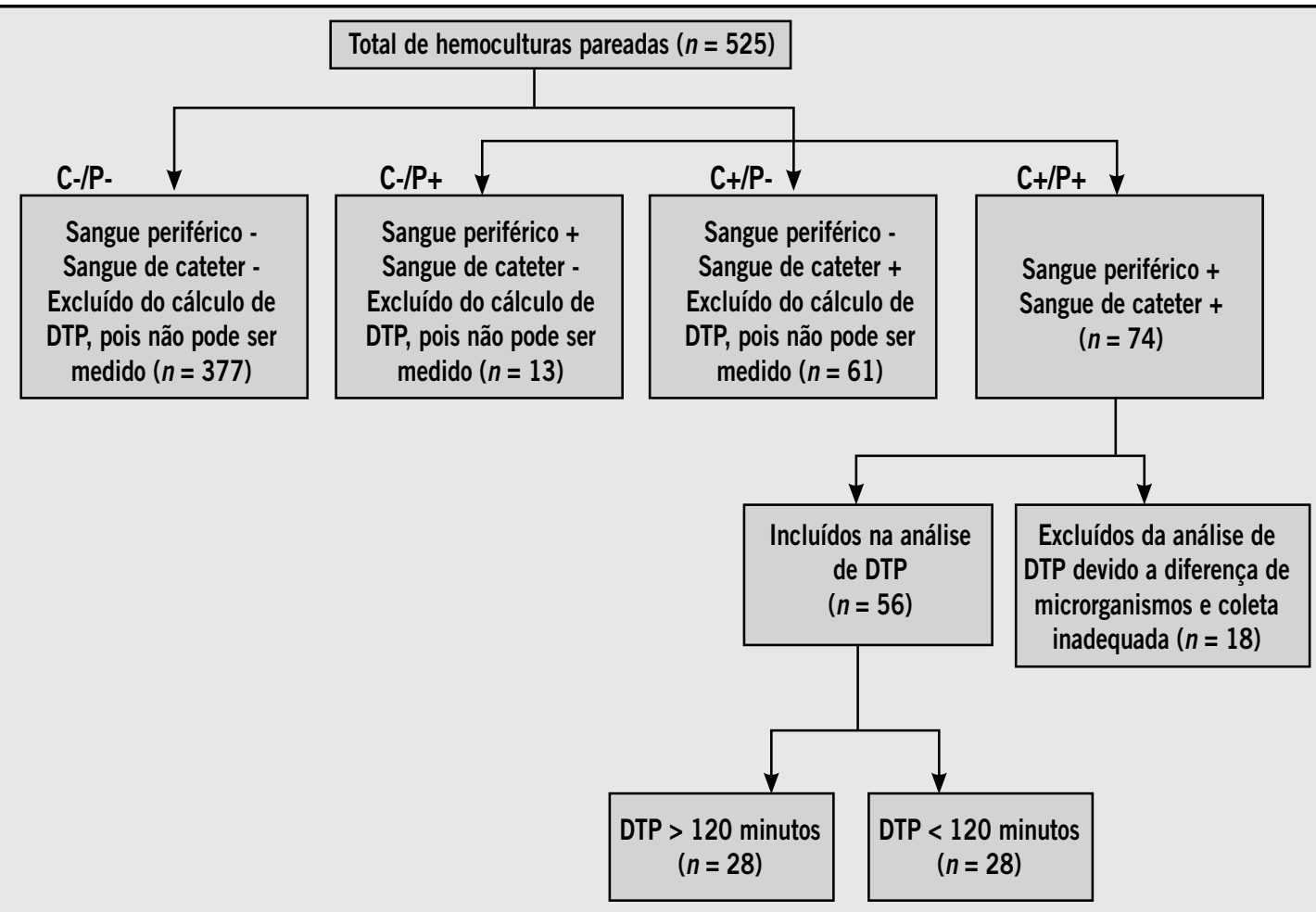

Figura 2 - Fluxograma das hemoculturas pareadas estudadas (HC/FMUSP, maio-agosto, 2008)

HC/FMUSP: Hospital das Clínicas da Faculdade de Medicina da Universidade de São Paulo; DTP: diferença de tempo de positividade; C-/P-: hemocultura pareada negativa para sangue de cateter e sangue periférico; $C$-/P+: hemocultura pareada positiva somente para sangue periférico; $C+/ P$-: hemocultura pareada positiva somente para sangue de cateter; C+/P+: hemocultura pareada positiva para sangue de cateter e sangue periférico. 


\section{Discussão}

As ICSRCs são associadas a taxas significativas de morbimortalidade e custos hospitalares elevados ${ }^{(8,11,17,30)}$. Em situações em que a associação da infecção com o cateter é questionável, a retirada do dispositivo intravascular não é de fácil decisão. Na suspeita de ICSRC, um método diagnóstico não invasivo sempre é mais interessante, pois, em caso negativo, há a possibilidade de poupar a retirada do cateter ${ }^{(1,3)}$.

Atualmente, a metodologia mais utilizada para diagnóstico de ICSRC é a cultura semiquantitativa de ponta de cateter associada à hemocultura qualitativa de sangue periférico ${ }^{(15)}$. Essa metodologia pode levar à remoção desnecessária do dispositivo intravascular em um número elevado de casos e pode confundir o diagnóstico de ICSRC em decorrência de culturas negativas, uma vez que nesse procedimento (técnica de Maki) só se avalia a parte externa do cateter e não há avaliação da via intraluminal que frequentemente está colonizada ${ }^{(4,9,10,16,21,22,30)}$.

A DTP tem sido utilizada para diagnóstico de ICSRC com a vantagem de não necessitar a remoção do cateter. O princípio dessa técnica baseia-se no fato de que se o CVC é a fonte infecciosa e o sangue colhido pelo dispositivo intravascular terá maior concentração de microrganismos do que o sangue periférico, seu TP será menor. Raad et al. ${ }^{(23)}$ publicaram um estudo no qual a DTP foi avaliada quanto a sua utilidade no diagnóstico de ICRSC em pacientes oncológicos, em que sensibilidade e especificidade para CVC de curta duração foi $81 \%$ e $92 \%$ e para CVC de longa duração foi $93 \%$ e $75 \%$ respectivamente ${ }^{(2,3,16,23,24)}$. Contudo, alguns autores relatam baixa especificidade e baixo valor preditivo negativo para essa técnica, que podem ser atribuídos a dois fatores: amostragem pequena e antibioticoterapia momentos antes da coleta das amostras pareadas ${ }^{(29)}$.

A acurácia da técnica DTP depende da coleta simultânea de sangue de cateter e sangue periférico em um intervalo máximo de 15 minutos (amostras pareadas) e do volume coletado, que deve ser igual em ambas as amostras, que devem ser prontamente transportadas para o laboratório, a fim de que possam ser inseridas nos equipamentos. A infusão de antimicrobianos por meio do acesso vascular pode prejudicar a interpretação da DTP, pois resultados falso-negativos podem ocorrer devido ao prolongamento do TP das hemoculturas coletadas por essa via ${ }^{(23)}$. Portanto, recomenda-se lavar com soro fisiológico o lúmen do CVC antes da coleta de sangue, diminuindo, assim, a concentração local do antibiótico.
É frequente a solicitação de avaliação da DTP pelos clínicos em nossa instituição, principalmente em amostras de pacientes oncológicos, porém a padronização pré-analítica deve ser mais bem enfatizada.

As hemoculturas coletadas de forma pareada (C/P) representaram apenas 5\% das hemoculturas aeróbias analisadas pela Seção de Microbiologia no período de estudo. A maioria das amostras pareadas analisadas foi negativa (72\%). A positividade somente em amostras de sangue de cateter ocorreu em apenas 61 (12\%) das amostras coletadas de forma pareada, indicando que a ICS poderia estar relacionada com a colonização intraluminal do CVC. Já nas amostras com positividade somente em sangue periférico, sugere-se infecção oriunda de outra fonte que não o $\mathrm{CVC}^{(3,23)}$.

Das 56 hemoculturas pareadas em que foi realizado cálculo de DTP, 50\% tiveram DTP $<120$ minutos. Nesses casos, possivelmente o CVC não seria a fonte da bacteremia e, portanto, o dispositivo intravascular não precisaria ser retirado se apenas o critério laboratorial fosse considerado.

Os microrganismos mais comumente isolados em pacientes com ICSRC são SCN e S. aureus, que podem refletir colonização da pele do paciente e das mãos dos profissionais de saúde. Os bacilos Gram-negativos mais isolados são Pseudomonas aeroginosa, Acinetobacter spp. e Stenotrophomonas maltophilia. Candida albicans e C. parapsilosis aparecem como as principais leveduras desse gênero na patogênese das ICSRCs, pois, além de colonizarem as mãos dos profissionais manipuladores, estão associadas a infusões de glicose e nutrição parenteral ${ }^{(6,9,16)}$.

Nas hemoculturas avaliadas com $\mathrm{C}+/ \mathrm{P}+$ em que o cálculo de DTP foi $<120$ minutos, os cocos Gram-positivos representaram $42 \%$, os bacilos Gram-negativos, $54 \%$ e leveduras, 4\%. Já nas hemoculturas $\mathrm{C}+/ \mathrm{P}+$ em que o cálculo de DTP foi $>120$ minutos, os cocos Gram-positivos representam $42 \%$, os bacilos Gram-negativos, $40 \%$ e as leveduras, $18 \%$, o que nos chamou atenção.

Quando somente uma das amostras foi positiva (C-/P+ ou $\mathrm{C}+$ (P-), a distribuição dos cocos Gram-positivos foi de $54 \%$ e $68 \%$, respectivamente.

A distribuição de microrganismos encontrada neste estudo é concordante com a frequentemente reportada na literatura, porém essas comparações tornam-se difíceis devido, principalmente, às características peculiares de cada instituição, sendo que em nossa casuística os pacientes oncológicos podem ter contribuído na maior prevalência de leveduras. 
Essa maior prevalência de Candida spp. é preocupante, visto que algumas espécies podem produzir slime, favorecendo, por meio do biofilme formado, proteção contra neutrófilos e dificultando a atividade dos antimicrobianos, levando à falha terapêutica de tratamento com drogas ${ }^{(30)}$. Raad et al. observaram que os pacientes com ICSRC apresentavam bacteremia por Staphylococcus spp. com maior frequência e Blot et al. relataram que o isolamento de Staphylococcus spp. e Candida albicans era altamente sugestivo de $\mathrm{IRC}^{(3,23)}$.

O cateter foi retirado em $69 \%$ dos pacientes com possível ICSRC, por meio da análise laboratorial, e em somente $22 \%$ dos casos houve homologia de crescimento entre os microrganismos das hemoculturas e da cultura de cateter. Esse baixo valor de concordância pode ser decorrente do uso de antimicrobianos, além das limitações inerentes à metodologia de Maki, em que somente a via extraluminal do cateter é avaliada, sendo que a via intraluminal está mais associada aos processos infecciosos em geral ${ }^{(8,9,16)}$. Interessante ressaltar que, nos casos em que não houve homologia de microrganismos isolados, o SCN foi positivo em todos eles, indicando possível contaminação da via extraluminal do dispositivo central. Outro aspecto relevante é a alta prevalência de patógenos resistentes aos antimicrobianos nas ICSRCs ${ }^{21,22,25)}$. Neste estudo, 75\% dos $S$. aureus eram oxacilina resistentes (estafilococo resistente à meticilina [MRSA]).

O presente estudo apresenta limitações inerentes a um estudo retrospectivo, principalmente em relação ao pequeno número de amostras pareadas disponíveis para análise da DTP, inviabilizando, portanto, uma análise estatística mais detalhada.

\section{Conclusão}

A determinação laboratorial da DTP como ferramenta auxiliar na diferenciação de ICSRC é viável e fácil de ser executada quando as hemoculturas são processadas por sistema automatizado, porém o processo de coleta deve ser rigidamente padronizado. A DTP pode trazer subsídios para minimizar a retirada precoce de cateteres, mas deve ser interpretada sempre em conjunto com o quadro clínico do paciente.

\section{Referências}

1. ACUNÃ, M. et al. Differential time to positivity and quantitative cultures noninvasive diagnosis of catheterrelated blood stream infection in children. Pediatr Infect Dis J, v. 27, n. 8, p. 681-5, 2008.

2. BLOT, F. et al. Diagnosis of catheter-related bacteremia: a prospective comparison of the time to positivity of hubblood versus peripheral-blood cultures. Lancet Infect Dis, v. 354, p. 1071-7, 1999.

3. BLOT, F. et al. Earlier positivity of central-venous versus peripheral-blood cultures is highly predictive of catheterrelated sepsis. J Clin Microbiol, v. 36, n. 1, p. 105-9, 1998.

4. BOUZA, E.; BURILlO, A.; MUNOZ, P. Catheter-related infections: diagnosis and intravascular treatment. Clin Microbiol Infect, v. 8, n. 5, p. 265-74, 2002.

5. CHATZINIKOLAU, I. et al. Prospective study of the value of quantitative culture of organisms from blood collected through central venous catheters in differentiating between contamination and bloodstream infection. J Clin Microbiol, v. 44, n. 5, p. 1834-5, 2006.

6. CHEN, W-T. et al. Improving diagnosis of central venous catheter-related bloodstream infection by using differential time to positivity as a hospital-wide approach at a cancer hospital. J Infect, v. 59, n. 5, p. 317-23, 2009.

7. CLINICAL and Laboratory Standards Institute, 2008.

8. CRUMP, J. A.; COLLIGNON, P. J. Intravascular catheterassociated infections. Eur J Clin Microbiol Infect Dis, v. 19, p. $1-8,2000$.
9. FERRETTI, G. et al. Catheter-related bloodstream infections, part I: pathogenesis, diagnosis, and management. Cancer Control, v. 9, n. 6, p. 513-23, 2002.

10. FILHO, A. B. et al. Sepse primária, relacionada ao cateter venoso central. Medicina, Ribeirão Preto, v. 31, p. 363-8, 1998.

11. FORTUN, J. Infecciones asociadas a dispositivos intravasculares utilizados para la terapia de infusión. Enferm Infecc Microbiol Clin, v. 26, n. 3, p. 168-74, 2005.

12. GARLAND, J. S. et al. Cohort study of the pathogenesis and molecular epidemiology of catheter-related bloodstream infection in neonates with peripherally inserted central venous catheters. Infect Control Hosp Epidemiol, v. 29, n. 3, p. 243-9, 2008.

13. JARDIM, J. M. Avaliação das práticas de prevenção e controle da infecção da corrente sanguínea associada ao cateter venoso central de curta permanência por meio de indicadores clínicos. Tese (Mestrado em Enfermagem na Saúde do adulto) - Escola de Enfermagem. São Paulo: Universidade de São Paulo, 2011.

14. LOBO, R. D. et al. Impact of an educational program and policy changes on decreasing catheter-associated bloodstream infections in a medical intensive care unit in Brazil. Am J Infect Control, v. 33, n. 2, p. 83-7, 2005.

15. MAKI, D. G.; WEISE, C. E.; SARAFIN, H. W. A semiquantitative culture method for identifying 
intravenous-catheter-related-infections. N Engl J Med, v. 296, n. 23, p. 1305-9, 1977.

16. MERMAL, L. A. et al. Guidelines for the management of intravascular catheter-related infections. Clin Infect Dis, v. 32, p. 1249-72, 2001.

17. MESIANO, E. R. A. B.; HAMANN, E. M. Infecções da corrente sanguínea em pacientes em uso de cateter venoso central em unidades de terapia intensiva. Revista Latino-Americana de Enfermagem, v. 15, n. 3, 2007. Disponível em: <http://www.eerp.usp.br/rlae>. Acesso em: 17 fev. 2010.

18. MURRAY, P. R. et al. Manual of clinical microbiology. $9^{\text {th }}$ ed. Washington, DC: ASMPress, 2007.

19. O' GRADY, N. P. et al. Guidelines for the prevention of intravascular catheter related infections. Clin Infect Dis, v. 35, p. 1281-307, 2002.

20. PAWAR, M. et al. Central venous catheter-related blood stream infections: incidence, risk factors, outcome and associated pathogens. J Cardiothorac Vasc Anesth, v. 18, n. 3, p. 304-8, 2004.

21. QUESADA, R. M. B. et al. Culturas de pontas de cateter venosos centrais e perfil de resistência aos antimicrobianos de uso clínico. Rev Bras Anal Clin, v. 37, n. 1, p. 45-8, 2005.

22. RAAD, I. Intravascular catheter-related infections. Lancet, v. 351, p. 893-8, 1998.
23. RAAD, I. et al. Differential time to positivity: a useful method for diagnosing catheter-related bloodstream infections. Ann Intern Med, v. 140, p. 18-25, 2004.

24. RAAD, I.; HANNA, H.; MAKI, D. Intravascular catheterrelated infections: advances in diagnosis, prevention and management. Lancet Infect Dis, v. 7, p. 645-57, 2007.

25. REACHER, M. H. et al. Bacteraemia and antibiotic resistance of its pathogens reported in England and Wales between 1990 and 1998: trend analysis. BMJ, v. 320, p. 213-6, 2000.

26. RESIC, H. et al. Prevalence of MRSA infections in patients on hemodialysis. Med Pregl, v. 60, n. 2, p. 97-100, 2007.

27. STORTI, A. et al. Biofilme detectado em ponta de cateter venoso central por cultura usando método quantitativo. Rev Bras Anal Clin, v. 39, n. 3, p. 183-7, 2007.

28. TARDIVO, T. B.; FARHAT NETO, J.; FARHAT FILHO, J. Infecções sanguíneas relacionadas aos cateteres venosos. Rev Bras Clin Med, v. 6, n. 6, p. 224-7, 2008.

29. VERWAEST, C. et al. Difference in time to positivity of hub-blood versus nonhub-blood culture is not useful for the diagnosis of catheter-related bloodstream infection in critically ill patients. Crit Care Med, v. 29, n. 7, p. 1399-403, 2001.

30. WORTHINGTON, T.; ELLIOTT, T. S. J. Diagnosis of central venous catheter related infection in adult patient. J Infect, v. 51, p. 267-80, 2005. 
Devido a erros de edição na Tabela Distribuição de microrganismos isolados de hemoculturas de acordo com origem (sangue de cateter e sangue periférico) e DTP, do artigo "Diferença de tempo de positividade: método útil no diagnóstico de infecção de corrente sanguínea relacionada com cateter?" de autoria de Karoline de Lemes Giuntini Corrêa; Flávia Rossi; João Nobrega de Almeida Júnior; e Gisele Madeira Duboc de Almeida, publicado no J Bras Patol Med Lab, v. 48, n. 3, p. 195-202, 2012, considerar a tabela abaixo em substituição à anterior.

\section{Tabela}

\begin{tabular}{|c|c|c|c|c|c|c|}
\hline \multirow{2}{*}{ Microrganismos } & \multicolumn{2}{|c|}{$\mathrm{C}_{+} / \mathrm{P}+$} & \multirow{2}{*}{$\begin{array}{c}\mathrm{C}+/ \mathrm{P}+ \\
\text { DTP não calculada* }\end{array}$} & \multirow[t]{2}{*}{$\mathrm{C}+/ \mathrm{P}-$} & \multirow[t]{2}{*}{$\mathrm{C}_{-} / \mathrm{P}_{+}$} & \multirow[t]{2}{*}{ Total } \\
\hline & DTP $<120$ & DTP $>120$ & & & & \\
\hline$n(\%)$ & $28(100)$ & $28(100)$ & $33(100)$ & $65(100)$ & $13(100)$ & $167(100)$ \\
\hline \multicolumn{7}{|c|}{ Gram-positivos } \\
\hline $\begin{array}{l}\text { Staphylococcus coagulase } \\
\text { negativo }\end{array}$ & $\varnothing$ & $2(7)$ & $6(18)$ & $33(51)$ & $4(31)$ & $45(28)$ \\
\hline Staphylococcus aureus & $8(28)$ & $6(22)$ & $7(22)$ & $4(6)$ & $2(17)$ & $27(16)$ \\
\hline Enterococcus spp. & $2(7)$ & $4(14)$ & $5(15)$ & $5(8)$ & $1(7)$ & $17(10)$ \\
\hline \multicolumn{7}{|c|}{ Gram-negativos } \\
\hline Pseudomonas aeruginosa & $3(11)$ & $\varnothing$ & $4(12)$ & $3(4)$ & $4(31)$ & $15(9)$ \\
\hline Acinetobacter spp. & $3(11)$ & $\varnothing$ & $3(9)$ & $\varnothing$ & $1(7)$ & $12(7)$ \\
\hline Escherichia coli & $2(7)$ & $\varnothing$ & $\varnothing$ & $2(3)$ & - & - \\
\hline Klebsiella spp. & $2(7)$ & $2(7)$ & $2(6)$ & $\varnothing$ & $1(7)$ & $4(4)$ \\
\hline Enterobacter spp. & $2(7)$ & $2(7)$ & $\varnothing$ & $\varnothing$ & $\varnothing$ & $6(3)$ \\
\hline Cryseobacterium spp. & $\varnothing$ & $2(7)$ & $2(6)$ & $\varnothing$ & $\varnothing$ & $7(4)$ \\
\hline Outros & $5(18)$ & $5(18)$ & $2(6)$ & $13(20)$ & - & $22(13)$ \\
\hline \multicolumn{7}{|c|}{ Levedura } \\
\hline Candida spp. & $1(3)$ & $5(18)$ & $2(6)$ & $1(2)$ & $\emptyset$ & $9(6)$ \\
\hline
\end{tabular}

